\title{
Article \\ Ancient Evolutionary History of Human Papillomavirus Type 16, 18 and 58 Variants Prevalent Exclusively in Japan
}

\author{
Kohsei Tanaka ${ }^{1,2}$, Gota Kogure ${ }^{1,3}$, Mamiko Onuki ${ }^{3}$, Koji Matsumoto ${ }^{3}$ (D), Takashi Iwata ${ }^{2}$, Daisuke Aoki ${ }^{2}$ (D) \\ and Iwao Kukimoto ${ }^{1, *}$ \\ 1 Pathogen Genomics Center, National Institute of Infectious Diseases, Tokyo 208-0011, Japan; \\ kohsei@nih.go.jp (K.T.); gota@nih.go.jp (G.K.) \\ 2 Department of Obstetrics and Gynecology, Keio University School of Medicine, Tokyo 160-0016, Japan; \\ iwatatakashi@1995.jukuin.keio.ac.jp (T.I.); aoki@z7.keio.jp (D.A.) \\ 3 Department of Obstetrics and Gynecology, Showa University School of Medicine, Tokyo 142-8666, Japan; \\ monuki@med.showa-u.ac.jp (M.O.); matsumok@mui.biglobe.ne.jp (K.M.) \\ * Correspondence: ikuki@niid.go.jp; Tel.: +81-42-561-0771
}

check for updates

Citation: Tanaka, K.; Kogure, G.; Onuki, M.; Matsumoto, K.; Iwata, T.; Aoki, D.; Kukimoto, I. Ancient Evolutionary History of Human Papillomavirus Type 16, 18 and 58 Variants Prevalent Exclusively in Japan. Viruses 2022, 14, 464 https://doi.org/10.3390/v14030464 Academic Editor: Tina Dalianis

Received: 4 February 2022

Accepted: 22 February 2022

Published: 24 February 2022

Publisher's Note: MDPI stays neutral with regard to jurisdictional claims in published maps and institutional affiliations.

Copyright: (C) 2022 by the authors. Licensee MDPI, Basel, Switzerland. This article is an open access article distributed under the terms and conditions of the Creative Commons Attribution (CC BY) license (https:// creativecommons.org/licenses/by/ $4.0 /$ )

\begin{abstract}
Human papillomavirus (HPV) is a sexually transmitted virus with an approximately 8-kilo base DNA genome, which establishes long-term persistent infection in anogenital tissues. High levels of genetic variations, including viral genotypes and intra-type variants, have been described for HPV genomes, together with geographical differences in the distribution of genotypes and variants Here, by employing a maximum likelihood method, we performed phylogenetic analyses of the complete genome sequences of HPV16, HPV18 and HPV58 available from GenBank $(n=627,146$ and 157 , respectively). We found several characteristic clusters that exclusively contain HPV genomes from Japan: two for HPV16 (sublineages A4 and A5), one for HPV18 (sublineage A1) and two for HPV58 (sublineages A1 and A2). Bayesian phylogenetic analyses of concatenated viral gene sequences showed that divergence of the most recent common ancestor of these Japan-specific clades was estimated to have occurred $~ 98,000$ years before present (YBP) for HPV16 A4, 39,000 YBP for HPV16 A5, 38,000 YBP for HPV18 A1, 26,000 for HPV58 A1 and 25,000 YBP for HPV58 A2. This estimated timeframe for the divergence of the Japan-specific clades suggests that the introduction of these HPV variants into the Japanese archipelago dates back to at least $\sim 25,000 \mathrm{YBP}$ and provides a scenario of virus co-migration with ancestral Japanese populations from continental Asia during the Upper Paleolithic period.
\end{abstract}

Keywords: human papillomavirus; Bayesian phylogenetics; most recent common ancestor; upper paleolithic era; Japanese archipelago

\section{Introduction}

Human papillomaviruses (HPVs) are a family of small non-enveloped viruses having a circular double-stranded DNA genome of approximately $8000 \mathrm{bp}$ [1]. More than 200 genotypes have been identified based on a $>10 \%$ difference in the $L 1$ capsid gene sequence [2]. HPVs infect basal epithelial cells in either cutaneous or mucosal tissues, and around 60 genotypes are phylogenetically clustered and recognized as mucosa-tropic genotypes [3]. Such HPVs are considered to be sexually transmitted, and at least 15 types are causatively associated with the development of human malignancies, including cervical, other anogenital, and head-and-neck cancers [4]. HPV16 is the most prevalent type detected in cervical cancer worldwide, followed by HPV18 [5]. Although HPV58 is the seventh most common type of cervical cancer across the world, it is the third most dominant type in East Asia, due to geographical variations of HPV type distribution.

Despite the existence of a large number of genotypes, HPV genome sequences are considered to be highly stable compared to RNA viruses because HPV genome replication 
relies completely on the high-fidelity DNA polymerases of the host cell [6]. Within individual genotypes, however, the HPV genome harbors another level of genetic complexity, called intra-type variants, which generally show less than $10 \%$ differences in the complete viral genome sequence. In this context, $1.0-10 \%$ and $0.5-1.0 \%$ nucleotide differences are defined as variant lineages and sublineages, respectively [7]. Indeed, a recent genomics study of HPV16 documented more than 2000 slightly different viral genomes from clinical samples [8]. Such high levels of minor genetic variations are most likely the results of viral genome editing or mutagenesis by host-cell cytosine deaminases of the APOBEC 3 family during the long evolutionary history of virus-host interaction [9-11].

The time scale of PV evolution over millions of years of co-evolution with their hosts is largely inferred from a model of virus-host co-divergence [12-14]. This is because vertebrate PVs exhibit strict host specificity for infection and the molecular phylogeny of PV genomic sequences shows a concordance with that of the host species, despite some incongruences being present [15]. Recently, regarding HPV genomic diversity, several studies have provided intriguing scenarios for the evolutionary history of HPVs. Firstly, by dating the divergence times of HPV16 variant lineages with a Bayesian statistics framework, Pimenoff et al. showed that HPV16 A and B/C/D variant lineages split apart 500,000 years before present (YBP), which largely predates the birth of Homo sapiens ( 200,000 YBP) and coincides with the timing of the split between Neanderthals and Homo sapiens [16]. Considering the geographic distribution of extant HPV16 variants (e.g., lineage A variants are exclusively prevalent in Eurasia, whereas lineage B variants are dominant only in Africa), these data support the Hominin-host-switch model, in which sexual transmission of the HPV16 A lineage from archaic to modern human populations occurred in Eurasia after out-of-Africa migration of Homo sapiens 90,000 YBP. To precisely annotate the divergence times of HPV16 variants, a subsequent study by Chen et al. established a molecular clock model inferred from PV sequences of non-human primates, which also estimated the first divergence of HPV16 variants to have occurred around 500,000 YBP [17]. Similar evolutionary timeframes of the radical divergence of major variant lineages were also reported for other HPV types [17,18]. Finally, recent studies implicated intra-host divergence following initial adaptation to specific niches, such as cutaneous or mucosal tissues, as a primary evolutionary pathway of human and non-human primate PVs, rather than strict virus-host co-divergence [18-20].

Although virus transmission from archaic humans is difficult to prove due to the lack of direct detection of HPV DNA in archaeological remains, virus co-migration with modern human populations after out-of-Africa migration seems to have had a significant impact on the present-day distributions of HPV genotypes and variants across the world $[16,17]$. Previously, we identified a genetic cluster of HPV16 variants that are exclusively prevalent in Japan, which we named sublineage A5 [21]. In the current study, we have comprehensively surveyed specific variants for HPV16, HPV18 and HPV58, and found similar Japan-specific clusters for all three genotypes. By dating the divergence times of these Japan-specific variants with a Bayesian Markov Chain Monte Carlo (MCMC) method, we discuss the evolutionary histories of these viruses based on the migration history of ancient Japanese ancestors.

\section{Materials and Methods}

\subsection{Viral Genome Sequences}

The complete genome sequences of HPV16 $(n=627)$, HPV18 $(n=146)$ and HPV58 $(n=157)$ genomes were retrieved from GenBank using the search terms "human papillomavirus type 16" [Primary Organism] OR "human papillomavirus type 18" [Primary Organism] OR "human papillomavirus type 58" [Primary Organism] AND complete genome [TITLE] (as of 31 August 2021) for phylogenetic analyses. Additionally, using next-generation sequencing approaches, as described previously [21], 49 complete genomic sequences of HPV16 were newly determined from cervical cancer and precancer specimens from women in Japan (the accession numbers are LC644164 to LC644191 and LC647437 to 
LC647457) and are included in this study. For comparison with HPV16 variants isolated from the United States, HPV16 sequences belonging to the sublineage A4 $(n=42)$ were extracted from a set of viral genome sequences reported by Mirabello et al. [8]; the list of accession numbers is shown in Table S1.

\subsection{Maximum Likelihood Phylogenetic Analyses}

For each genotype, virus complete genomic sequences including representative sequences for variant lineages/sublineages were aligned against each other using MAFFT v7.309 with default parameters. Maximum likelihood trees were inferred using RAxML v8.2.9 under the general time-reversible nucleotide model with gamma-distributed rate heterogeneity and invariant sites $(\mathrm{GTR}+\mathrm{G}+\mathrm{I})$, employing 1000 bootstrap replicates. Phylogenetic trees were visualized using FigTree v1.4.2 (http:/ / tree.bio.ed.ac.uk/software/figtree/, accessed on 31 August 2021).

\subsection{Bayesian Phylogenetic Analyses}

A Bayesian MCMC framework implemented by BEAST v2.6.5 was used to estimate the divergence time of the most recent common ancestor (MRCA) for HPV16/18/58 variants. Appropriate clock and tree models were determined for our datasets by the pass sampling method using the Path sampler in the BEAST2 packages, leading to selection of relaxed lognormal molecular clock and coalescent Bayesian skyline models. The concatenated nucleotide sequences of six open-reading frames $(E 6, E 7, E 1, E 2, L 2$ and $L 1)$ and a previously reported HPV16 evolutionary rate of $1.84 \times 10^{-8}$ substitutions/site/year [16] were used for all MCMC analyses. The most appropriate substitution models were determined by the best-fit model approach of jModelTest v2.1; the selected models were GTR + I + G for HPV16, TPM1uf + I for HPV18 and TVM + I + G for HPV58. The MCMC analysis was run for 100,000,000 chains, with sampling every 10,000 generations. After the first 10\% of the chain was omitted, effective sample sizes (ESS) were monitored by Tracer v1.7.1 and ESS greater than 200 were accepted. Maximum clade credibility trees were constructed using Tree Annotator v2.6.0, and the MCMC phylogenetic trees were visualized using FigTree v1.4.2.

\section{Results}

\subsection{Japan-Specific Clusters of HPV16/18/58 Genomes}

Thus far, we have determined a number of complete genomic sequences of HPV16 $(n=172)$, HPV18 $(n=21)$ and HPV58 $(n=57)$ from cervical swab samples of Japanese women [21-23]. To compare phylogenetic relationships of these Japanese isolates with HPV isolates reported worldwide, we used a maximum likelihood framework to construct phylogenetic trees of whole viral genomes.

The phylogenetic tree of a total of 627 complete genomes of HPV16 showed that the Japanese isolates were distributed across the lineages A, C and D (Figure 1), but most of them belonged to the lineage A $(161 / 172,93.6 \%)$, in particular the sublineage A4 (88/172, $51.2 \%$ ), which had originally been designated as the Asian lineage. Some of the Japanese isolates were also clustered into the clade of the sublineage A5 (28/172, 16.3\%) and, as we previously reported [21], this A5 cluster contained HPV16 genomes exclusively from Japan. Regarding the A4 variants from Japan, about one-half (41/88, 46.6\%) were clearly separated from the clade that included the reference A4 genome near the root of the tree. To determine whether this A4 cluster was unique to the HPV16 genomes from Japan, we accessed additional A4 genome sequences $(n=42)$ from a recent study of HPV16 in the United States [8], and performed phylogenetic analysis with these. It is shown in Figure S1, with one exception (accession number, MG848021); none of the US isolates were grouped into this cluster, indicating regional exclusivity or specificity of this A4 cluster harboring the Japanese isolates. 


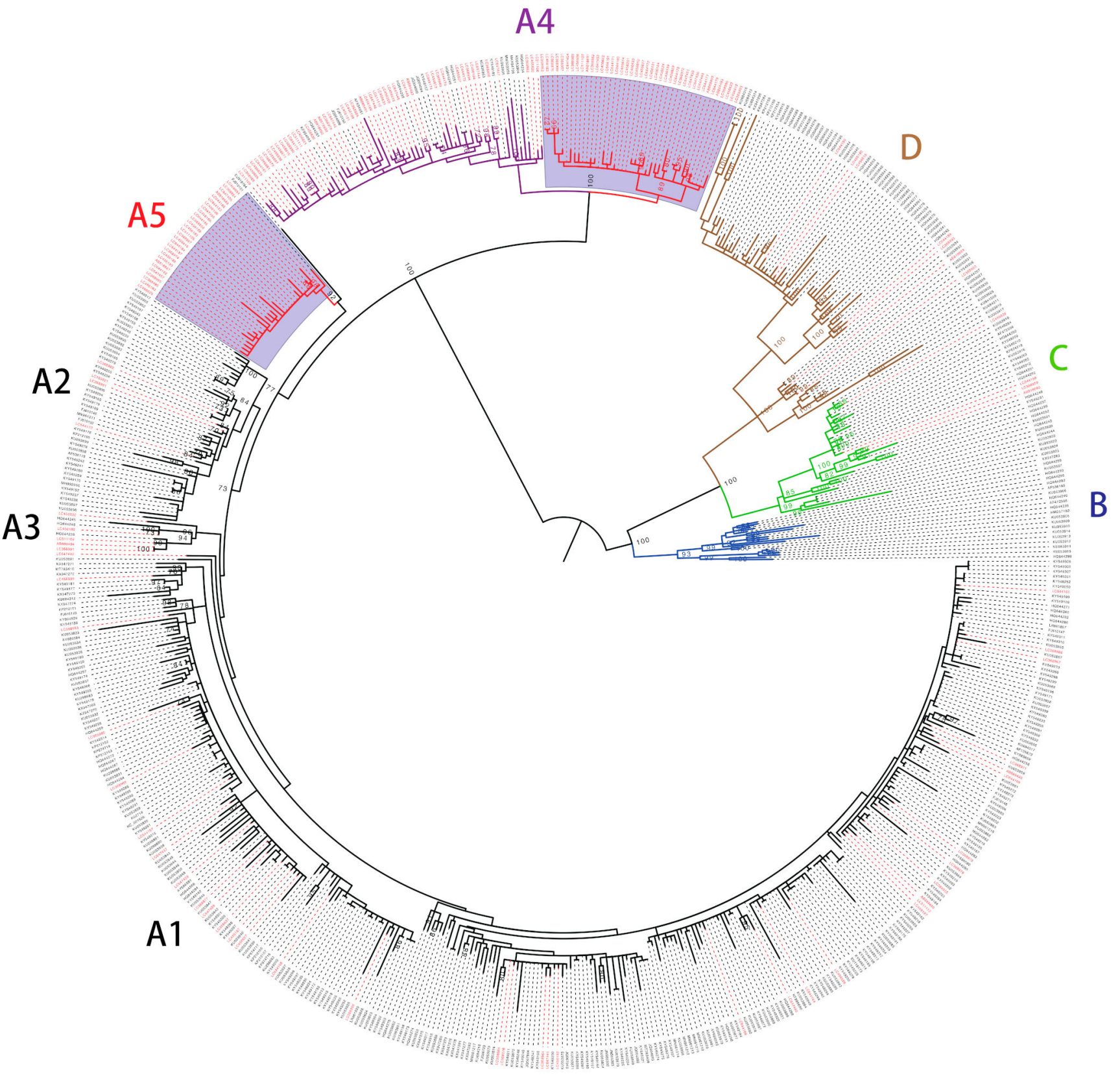

Figure 1. Maximum likelihood phylogenetic tree of complete HPV16 genomes available from GenBank (https: / / www.ncbi.nlm.nih.gov/genbank/, accessed on 31 August 2021). A total of 627 sequences were analyzed in RAxML with 1000 bootstrap replicates. Bootstrap values $>70 \%$ are displayed. Red taxa indicate 172 sequences from Japan. Purple area marks the Japan-specific cluster of the sublineage A4 and the cluster of the sublineage A5. Scale bar, nucleotide substitutions per site.

The phylogenetic tree of a total of 146 complete genome sequences of HPV18 revealed that most of the HPV18 isolates from Japan belonged to the sublineage A1 (19/21,90.5\%) (Figure 2). Among the A1 genomes from Japan, some (7/21,33.3\%) formed a cluster distinct from other A1 genomes reported across the world, suggesting the presence of Japan-specific HPV18 variants. 


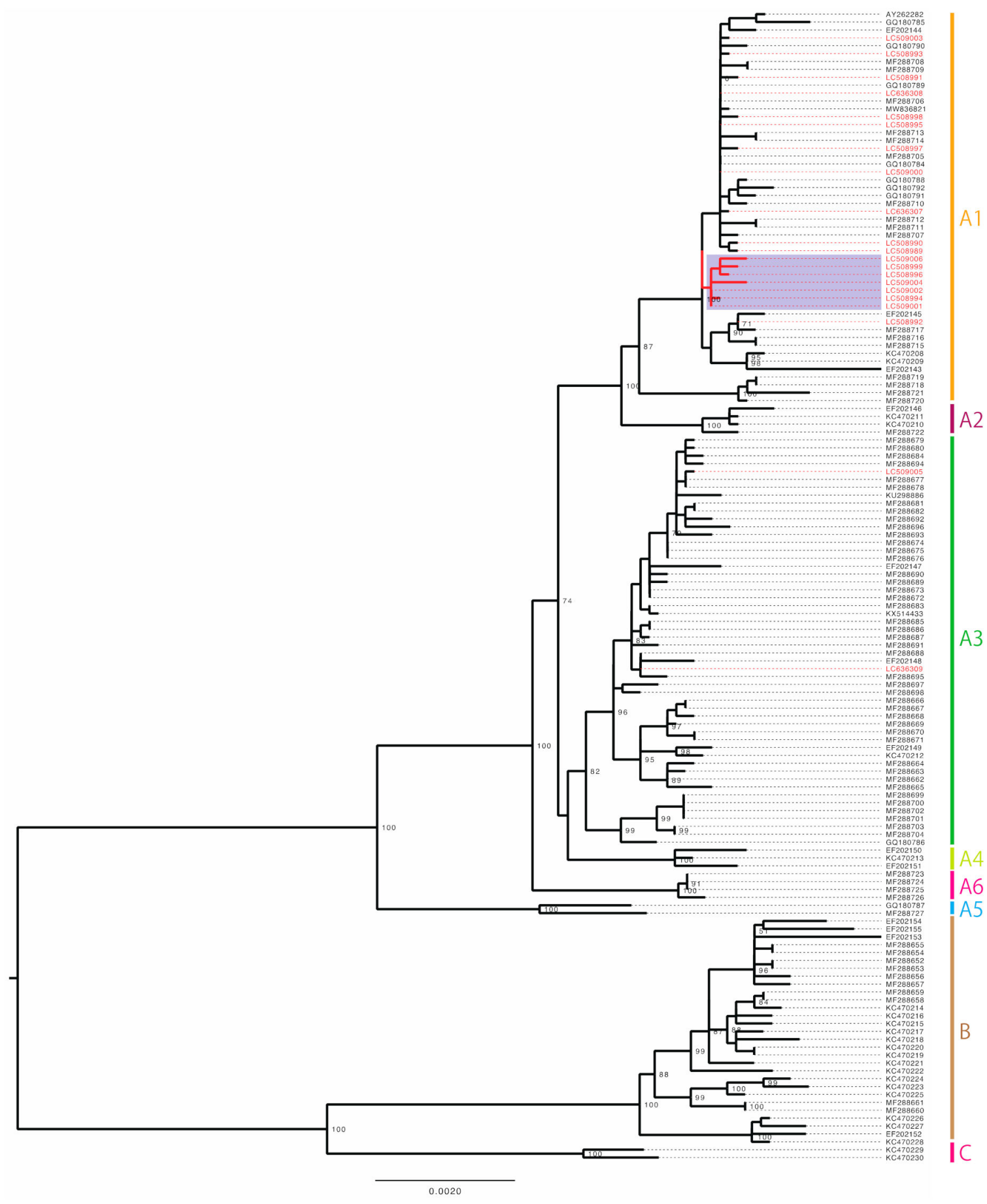

Figure 2. Maximum likelihood phylogenetic tree of complete HPV18 genomes available from GenBank (https: / /www.ncbi.nlm.nih.gov/genbank/, accessed on 31 August 2021). A total of 146 sequences were analyzed in RAxML with 1000 bootstrap replicates. Bootstrap values $>70 \%$ are displayed. Red taxa indicate 21 sequences from Japan. Purple area marks the Japan-specific cluster of the sublineage A1. Scale bar, nucleotide substitutions per site.

The phylogenetic tree of a total of 157 complete genome sequences of HPV58 showed that these genomes from Japan were widely distributed across the sublineages A1, A2 and A3 (Figure 3). Again, a cluster of the sublineage A1 that only contained the Japanese isolates $(13 / 57,22.8 \%)$, and a smaller cluster of the sublineage A2 that was enriched for the HPV58 genomes from Japan (7/57, 12.3\%), were found for HPV58, indicating that distinct exclusively Japanese clades were present for all three genotypes. 


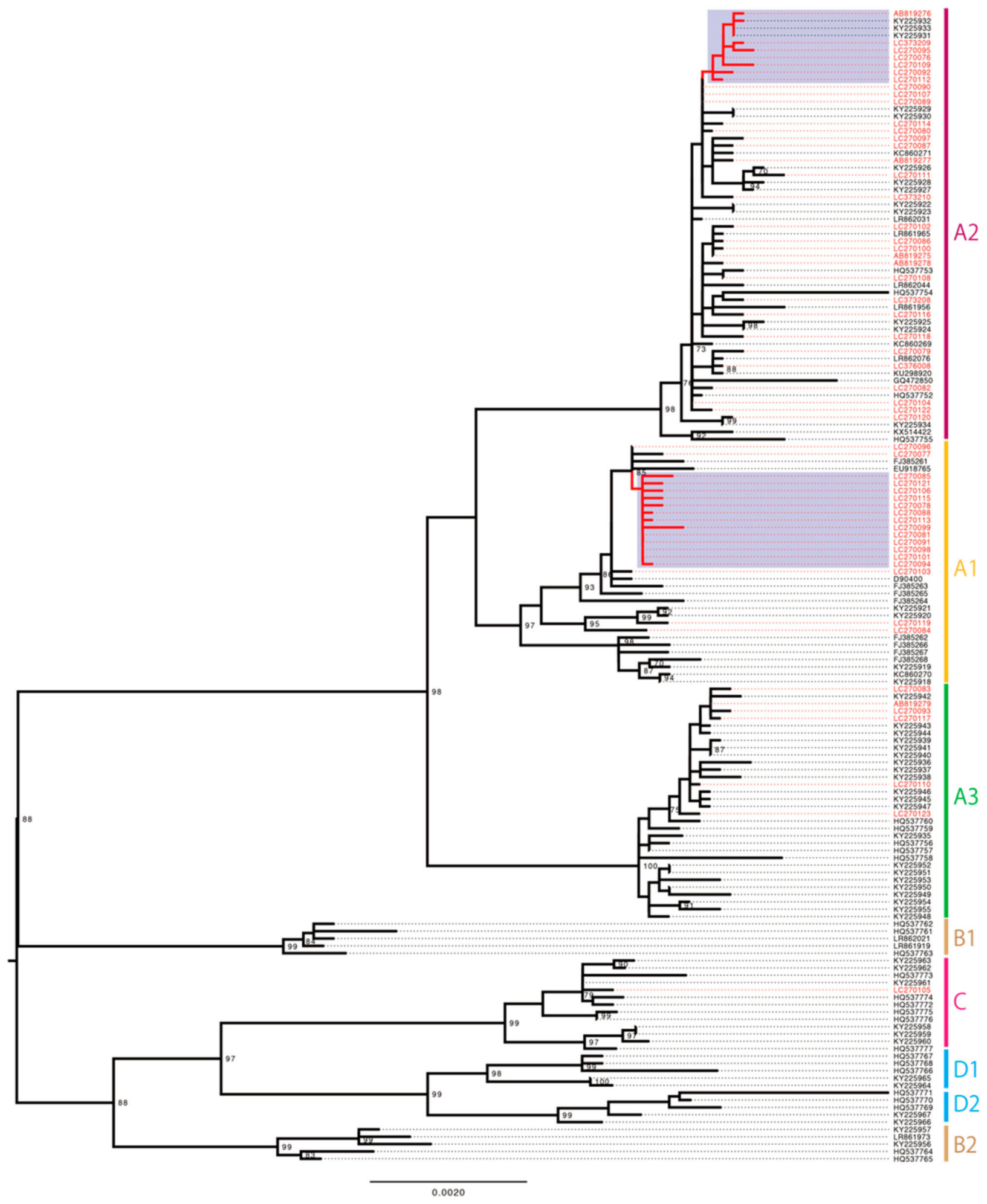

Figure 3. Maximum likelihood phylogenetic tree of complete HPV58 genomes available from GenBank (https: / /www.ncbi.nlm.nih.gov/genbank/, accessed date: 31 August 2021). A total of 157 sequences were analyzed in RAxML with 1000 bootstrap replicates. Bootstrap values $>70 \%$ are displayed. Red taxa indicate 57 sequences from Japan. Purple area marks the Japan-specific cluster of the sublineages A1 and A2. Scale bar, nucleotide substitutions per site.

\subsection{Estimation of the Divergence Time of Japan-Specific HPV16/18/58 Variants}

To estimate the evolutionary timeframe of the emergence of the Japan-specific HPV16/18/58 genomes, we employed a Bayesian phylogenetic framework for subse- 
quent analyses. The relaxed lognormal molecular clock and coalescent Bayesian skyline models, which were validated for HPV genome evolution in recent studies [16,17], were used with the concatenated virus gene sequences of E6,E7, E1, E2, L2 and L1.

The time-scaled maximum clade credibility tree of HPV16 showed a similar tree topology to the maximum likelihood tree (Figure 4). The deepest divergence between the lineage $\mathrm{A}$ and $\mathrm{B} / \mathrm{C} / \mathrm{D}$ was calculated to be 510,349 YBP (95\% highest posterior density [HPD] interval: 260,852-801,328), which was consistent with the results of previous studies, which were 461,000 YBP [16] and 488,900 YBP [17]. As shown in Table 1, the divergence times of the MRCAs were estimated to be 39,279 YBP (95\% HPD interval: 21,572-63,467) for the Japan-specific A5 variants and 97,091 YBP (95\% HPD interval: 61,272-136,486) for the Japan-specific A4 variants.

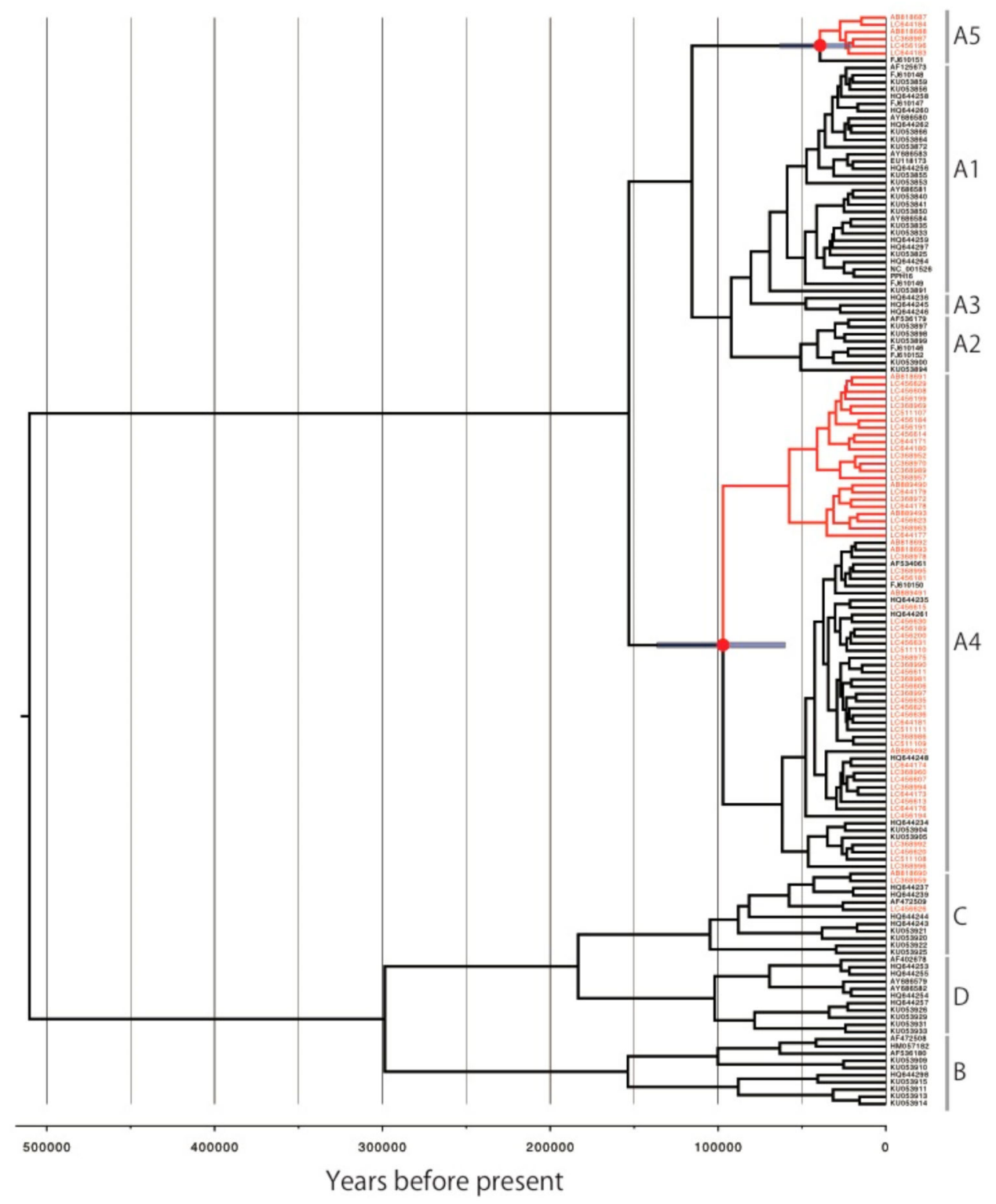

Figure 4. Bayesian MCMC phylogenetic tree of HPV16. The concatenated E6,E7,E1,E2, L2 and L1 sequences from 152 HPV16 genomes were analyzed in BEAST2. Time-scaled maximum-clade credibility tree is shown. Red node indicates the position of the most recent common ancestor for the Japan-specific HPV16 variants. Blue bar indicates 95\% highest posterior density interval.

The HPV16 sequence dataset of Pimenoff et al. [16] included seven HPV16 isolates from Japan, which was used to confirm our divergence time estimates. The Bayesian 
phylogenetic analysis of this dataset reconstructed a similar timeframe for HPV16 variant evolution as in the previous study (Figure S2). The divergence time of the MRCA for two Japanese A5 isolates (AB818687 and AB818688) and a Thai isolate (FJ610151) was estimated to be 39,681 YBP (95\% HPD interval: 20,423-64,452) (Table 1). One Japanese isolate (AB818691), belonging to the Japan-specific A4 cluster in our current study, diverged from the other A4 variants at 103,650 YBP (95\% HPD interval: 66,397-144,197).

For HPV18, an MCMC tree with a similar topology to the maximum likelihood tree was also reconstructed (Figure 5). The deepest divergence between the lineages $\mathrm{A}$ and $\mathrm{B} / \mathrm{C} / \mathrm{D}$ was estimated to be 543,988 YBP (95\% HPD interval: 339,460-775,927), consistent with 552,100 YBP result of a previous study [17]. The divergence time of the MRCA of the Japan-specific A1 variants was estimated to be 37,702 YBP (95\% HPD interval: 26,348-50,678) (Table 1).

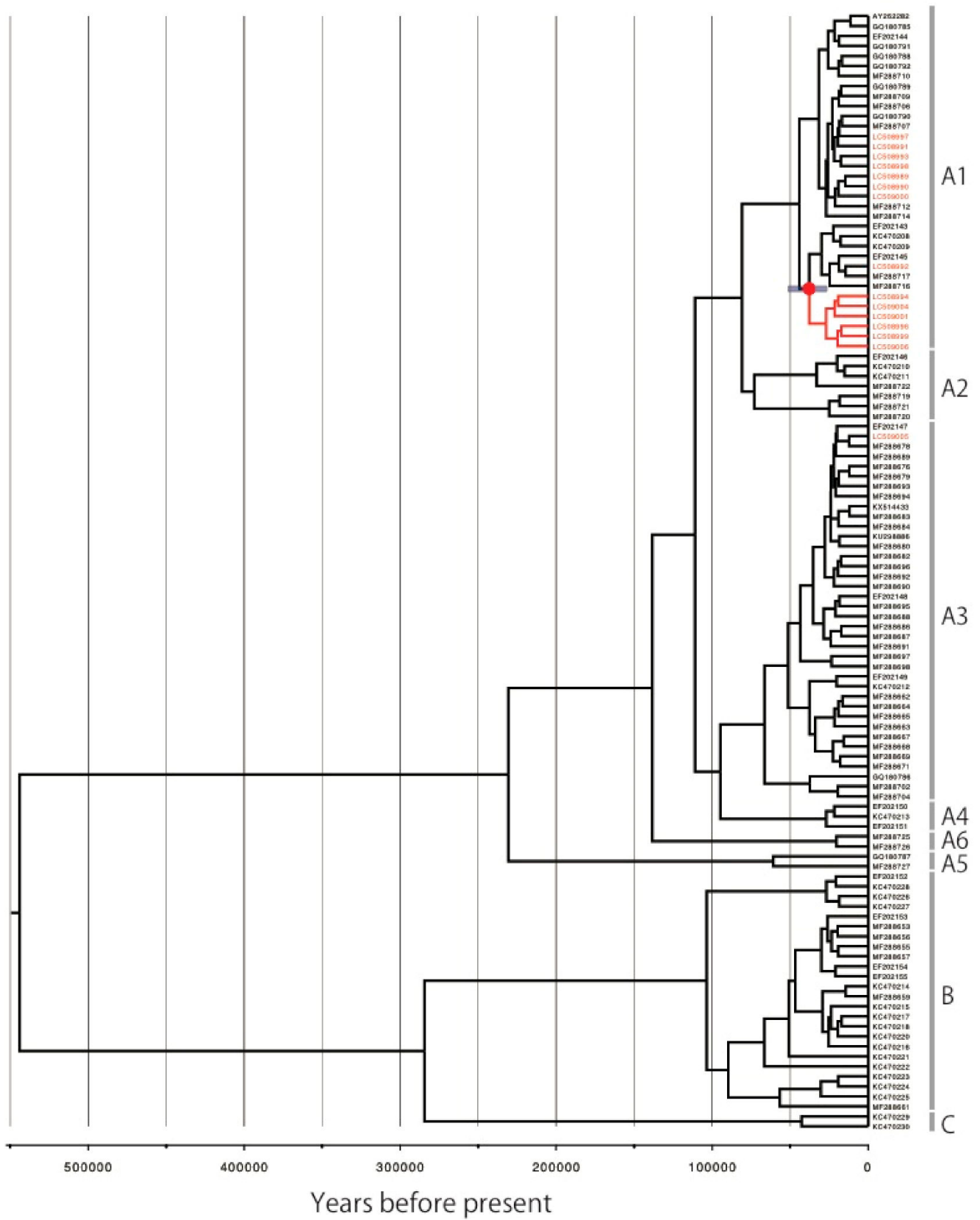

Figure 5. Bayesian MCMC phylogenetic tree of HPV18. The concatenated E6, E7,E1, E2, L2 and L1 sequences from 112 HPV18 genomes were analyzed in BEAST2. Time-scaled maximum-clade credibility tree is shown. Red node indicates the position of the most recent common ancestor for the Japan-specific HPV18 variants. Blue bar indicates 95\% highest posterior density interval. 
For HPV58, the MCMC tree also showed a topology consistent with the maximum likelihood tree (Figure 6). The deepest divergence between the lineages A and B/C/D was estimated to be 412,795 YBP (95\% HPD interval: 247,936-603,724), also consistent with the result of Chen et al., which was 478,600 YBP [18]. The divergence times of the MRCAs were estimated to be 25,879 YBP (95\% HPD interval: 17,403-30,208) for the Japan-specific A1 variants and 25,032 YBP (95\% HPD interval: 16,461-29,031) for the Japan-specific A2 variants (Table 1 ).

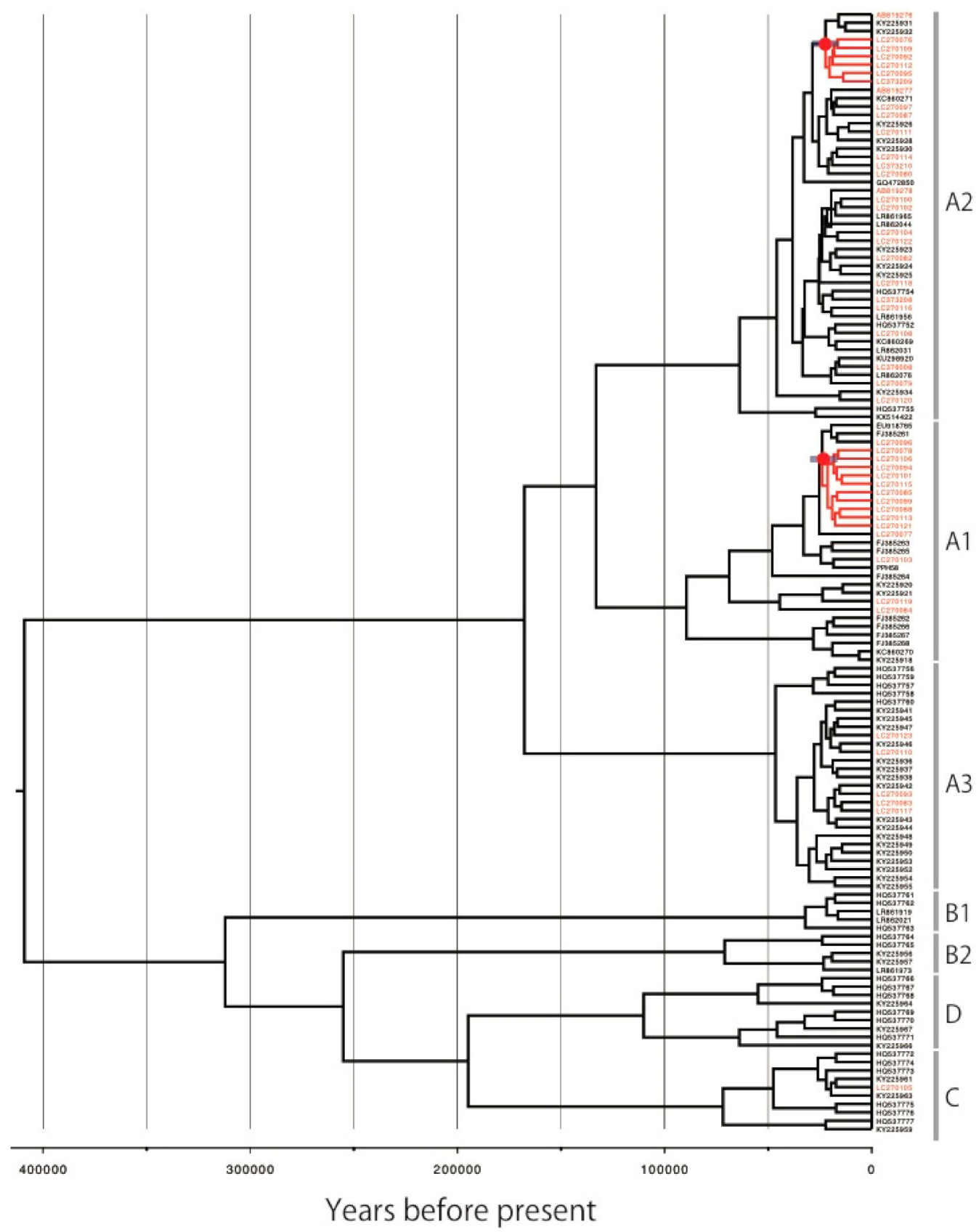

Figure 6. Bayesian MCMC phylogenetic tree of HPV58. The concatenated E6,E7,E1,E2, L2 and L1 sequences from 134 HPV58 genomes were analyzed in BEAST2. Time-scaled maximum-clade credibility tree is shown. Red node indicates the position of the most recent common ancestor for the Japan-specific HPV58 variants. Blue bar indicates 95\% highest posterior density interval. 
Table 1. Divergence time estimates of MRCAs of the Japan-specific HPV variants.

\begin{tabular}{cccc}
\hline Type & \multicolumn{1}{c}{ Variant } & $\begin{array}{c}\text { Divergence Time } \\
\text { (Years Ago) }\end{array}$ & 95\% HPD Interval \\
\hline HPV16 & Japan A5 vs. FJ610151 & 39,279 & $21,572-63,467$ \\
HPV16 & Japan A4 vs. Other A4 & 97,091 & $61,272-136,486$ \\
HPV16 * & Japan A5 vs. FJ610151 & 39,681 & $20,423-64,452$ \\
HPV16 * & Japan A4 vs. Other A4 & 103,650 & $66,397-144,197$ \\
HPV18 & Japan A1 vs. Other A1 & 37,702 & $26,348-50,678$ \\
HPV58 & Japan A1 vs. Other A1 & 25,879 & $17,403-30,208$ \\
HPV58 & Japan A2 vs. Other A2 & 25,032 & $16,461-29,031$ \\
\hline
\end{tabular}

*, the dataset of Pimenoff et al. (118 complete genomes) was used. Divergence time estimates are shown as mean values. HPD, highest posterior density.

\section{Discussion}

In this study, we explored the phylogenetic relationships discernable from the complete sequences of HPV16/18/58 genomes reported worldwide. We found characteristic genetic clusters that exclusively contain HPV variants from Japan in each of these three genotypes. These clusters belonged to the sublineages A4 and A5 for HPV16, the sublineage A1 for HPV18, and the sublineages A1 and A2 for HPV58. We then performed the Bayesian phylogenetic analyses to infer the evolutionary timeframe for the emergence of these Japan-specific variants, and showed that except for HPV16 A4 the divergence time of the MRCAs of these variants dated back to between 40,000-25,000 YBP, corresponding to the Upper Paleolithic era. How does this estimated timeframe explain the emergence of the Japan-specific HPV variants in relation to the history of the ancient Japanese?

The Japanese archipelago is located east of the Asian continent and has seen human activity since at least 30,000 YBP. Regarding the origin of the current Japanese, two prehistoric overseas migrations are considered to have contributed to the formation of the ancestral Japanese population (Figure 7) [24]. One of these was the first waves of migration from mainland Asia, which are supposed to have occurred during the late Pleistocene between 40,000-30,000 YBP, based on archaeological evidence such as stone tools and the oldest human remains dating back to $\sim 36,500$ YBP [25]. This Upper Paleolithic period was followed by the Jomon period that lasted from 16,500 to 3000 YBP, lived by the Jomon people with a hunter-gatherer lifestyle. The second migration events from Northeast Asia occurred after 3000 YBP in the Holocene, together with the introduction of rice farming into the Japanese archipelago, which marks the start of the Yayoi period. Recent genome-wide single-nucleotide polymorphisms studies of the current and ancient Japanese [26-29] support the dual structure model, in which the Yayoi migrants interbred with the indigenous Jomon people [30,31], as a model for the origin of the present-day Japanese.

\begin{tabular}{|l|l|l|}
\hline Upper Paleolithic & Jomon & Yayoi \\
\hline
\end{tabular}

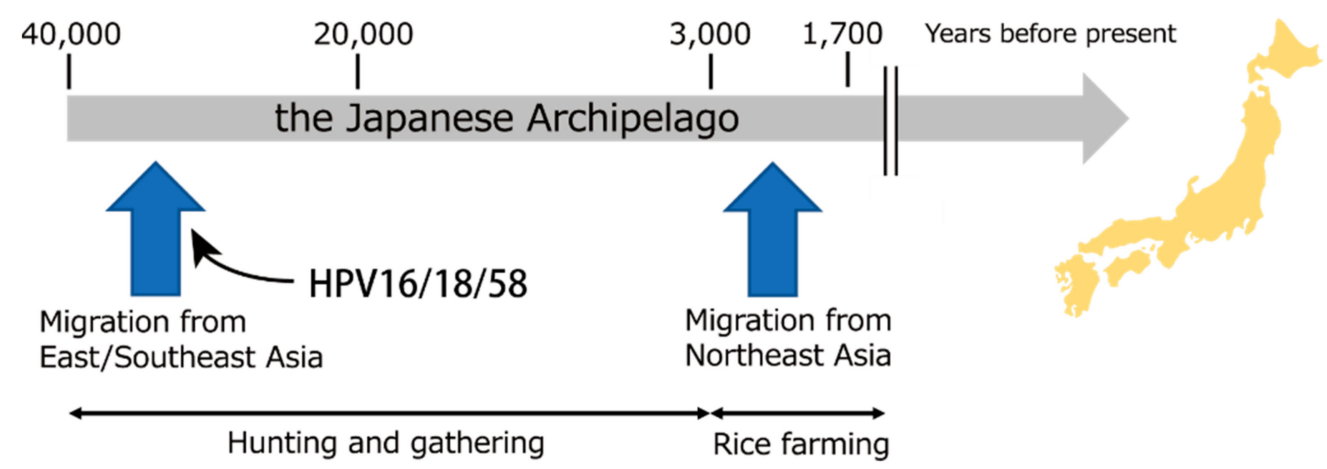

Figure 7. Timeframe of migration of ancestral Japanese populations into the Japanese archipelago and co-migration of Japan-specific HPV16/18/58 variants. 
Considering these population histories, our estimated divergence times of the MRCAs of the Japan-specific HPV16/18/58 genomes imply that these variants were introduced into the Japanese archipelago, most probably with the first migration at 40,000-25,000 YBP (Figure 7). Although the population continuity between the Paleolithic and Jomon periods remains elusive, we speculate that these HPV variants were spread via sexual transmission within the prehistoric Japanese population in the Upper Paleolithic era and subsequently passed on to the Jomon people, a significant source of genetic ancestry of the current Japanese.

In contrast, the deep divergence of the Japan-specific HPV16 A4 variants from other A4 variants ( 97,000 YBP) indicates that these variants were generated much earlier than other Japan-specific variants. Recent studies investigating the divergence time of HPV16 variants suggest that the major split of $A 4$ variants from the $A 1 / 2 / 3$ variants occurred 100,000 YBP [16] and 200,000 YBP [17]. This suggests transmission of A4 variants from archaic hominins, such as Denisovans, to Homo sapiens in East Eurasia, which explains the exclusive prevalence of A4 variants in this region today. The deep divergence observed for the Japan-specific A4 variants in our study may also reflect such viral transmission from archaic hominins to Japanese ancestors in continental Asia, followed by co-migration of these variants into the Japanese archipelago. A recent study on ancient DNA from Jomon individuals indicated that the Jomon lineage diverged before the diversification of presentday East Eurasian populations, suggesting that the Jomon is one of the oldest lineages in East Eurasia [28,29]. Thus, the ancestral population of the Jomon lineage in East Eurasia might have introduced the Japan-specific A4 variants into the Japanese archipelago, without leaving any trace of such variants in the place from which they departed. Alternatively, because the HPV16 genomes analyzed in our study included only seven isolates from China and five from Thailand, HPV16 variants that are closely related to the Japan-specific A4 variants might remain undiscovered in East/Southeast Asia. Such yet-unexplored variants may fill the gap of the evolutionary timeframe of the Japan-specific A4 variants and provide further insight into the evolution of HPV16 variants.

Our scenario that particular HPV variants have been continuously present within the Japanese population for at least $\sim 25,000$ years might be surprising, but also conceivable given that sexual transmission is one of the best strategies for a virus to sustain its infection cycles from generation to generation, and that the Japanese archipelago is geographically isolated and experienced no large-scale migration events after the Yayoi period. Another important factor that might facilitate the maintenance of specific HPV variants within a population is viral adaptation to that particular population. In this regard, the Japanspecific HPV16 A5 genomes encode a characteristic E2 protein, A105T, not seen in other variant genomes [21]. The E2 protein is a viral replication/transcription factor and is required for stable maintenance of virus genomes through cell division [32]. E2 expression in cervical basal cells is considered to be critical for long-term persistent infection [33], and its immune recognition by cytotoxic T-lymphocytes (CTL) likely contributes to clearing HPV infections [34,35]. Thus, the A105T variant may assist immune evasion by changing the CTL epitopes in the E2 protein. For instance, if these altered CTL epitopes are not optimal, particularly in Japanese people, this could facilitate long-term viral persistence. Because E2 amino acid changes are also observed for the Japan-specific HPV58 A1 variants (i.e., S275N), further studies should be focused on the immunological properties of this viral protein.

\section{Conclusions}

This study has revealed the ancient history of Japan-specific HPV variants, strongly suggesting virus co-migration with prehistoric Japanese ancestors into the Japanese archipelago. Given an extremely slow rate of HPV evolution, it can be envisioned that other regions of the world have also kept their own HPV variants within their populations, and that such HPV variants may provide novel insights into a yet-to-be-defined history of human populations. 
Supplementary Materials: The following are available online at https:/ /www.mdpi.com/article/10 $.3390 / v 14030464 /$ s1, Figure S1: Maximum likelihood phylogenetic tree of HPV16 genome sequences including 42 A4 genome sequences from Mirabello et al., Figure S2: Bayesian MCMC phylogenetic tree of 118 HPV16 genome sequences from Pimenoff et al., Table S1: HPV16 genomic sequences of the A4 sublineage retrieved from Mirabello et al.

Author Contributions: Conceptualization, I.K.; formal analysis, K.T., I.K.; investigation, K.T., G.K.; resources, M.O., K.M., T.I.; data curation, K.T.; writing-original draft preparation, I.K.; writingreview and editing, K.T., I.K.; supervision, D.A.; funding acquisition, M.O., K.M., I.K. All authors have read and agreed to the published version of the manuscript.

Funding: This study was supported by Grant-in-Aid for Scientific Research (C) (grant numbers JP20K09682 to I.K. and JP20K09677 to M.O.) and Grant-in-Aid from the Japan Agency for Medical Research and Development (grant numbers JP21fk0108098 to I.K., K.M. and JP21fk0108102 to I.K.).

Institutional Review Board Statement: The study was conducted in accordance with the Declaration of Helsinki, and approved by the institutional ethical and research review board of National Institute of Infectious Diseases (protocol code 1042; date of approval, 3 September 2019).

Informed Consent Statement: Informed consent was obtained from all subjects involved in the study.

Data Availability Statement: All HPV genome sequences used are available from the GenBank database.

Acknowledgments: We thank Tsuyoshi Sekizuka (National Institute of Infectious Diseases, Japan) for instructing us in the Bayesian MCMC analyses methods and Hideaki Kanzawa (National Museum of Nature and Science, Japan) for valuable comments on the manuscript.

Conflicts of Interest: The authors declare no conflict of interest.

\section{References}

1. Doorbar, J.; Egawa, N.; Griffin, H.; Kranjec, C.; Murakami, I. Human papillomavirus molecular biology and disease association. Rev. Med. Virol. 2015, 25 (Suppl. 1), 2-23. [CrossRef]

2. Bzhalava, D.; Eklund, C.; Dillner, J. International standardization and classification of human papillomavirus types. Virology 2015, 476, 341-344. [CrossRef]

3. Burk, R.D.; Chen, Z.; Van Doorslaer, K. Human Papillomaviruses: Genetic Basis of Carcinogenicity. Public Health Genom. 2009, 12, 281-290. [CrossRef] [PubMed]

4. Arbyn, M.; Tommasino, M.; Depuydt, C.; Dillner, J. Are 20 human papillomavirus types causing cervical cancer? J. Pathol. 2014, 234, 431-435. [CrossRef] [PubMed]

5. De Sanjose, S.; Quint, W.G.; Alemany, L.; Geraets, D.T.; Klaustermeier, J.E.; Lloveras, B.; Tous, S.; Felix, A.; Bravo, L.E.; Shin, H.R.; et al. Human papillomavirus genotype attribution in invasive cervical cancer: A retrospective cross-sectional worldwide study. Lancet Oncol. 2010, 11, 1048-1056. [CrossRef]

6. McBride, A.A. Replication and Partitioning of Papillomavirus Genomes. Adv. Virus Res. 2008, 72, 155-205. [CrossRef]

7. Burk, R.D.; Harari, A.; Chen, Z. Human papillomavirus genome variants. Virology 2013, 445, 232-243. [CrossRef]

8. Mirabello, L.; Yeager, M.; Yu, K.; Clifford, G.M.; Xiao, Y.; Zhu, B.; Cullen, M.; Boland, J.F.; Wentzensen, N.; Nelson, C.; et al. HPV16 E7 Genetic Conservation Is Critical to Carcinogenesis. Cell 2017, 170, 1164-1174.e6. [CrossRef] [PubMed]

9. Hirose, Y.; Onuki, M.; Tenjimbayashi, Y.; Mori, S.; Ishii, Y.; Takeuchi, T.; Tasaka, N.; Satoh, T.; Morisada, T.; Iwata, T.; et al. Within-Host Variations of Human Papillomavirus Reveal APOBEC Signature Mutagenesis in the Viral Genome. J. Virol. 2018, 92, e00017-18. [CrossRef] [PubMed]

10. Zhu, B.; Xiao, Y.; Yeager, M.; Clifford, G.; Wentzensen, N.; Cullen, M.; Boland, J.F.; Bass, S.; Steinberg, M.K.; Raine-Bennett, T.; et al. Mutations in the HPV16 genome induced by APOBEC3 are associated with viral clearance. Nat. Commun. 2020, 11, 886. [CrossRef] [PubMed]

11. Warren, C.; Van Doorslaer, K.; Pandey, A.; Espinosa, J.M.; Pyeon, D. Role of the host restriction factor APOBEC3 on papillomavirus evolution. Virus Evol. 2015, 1, vev015. [CrossRef]

12. Gottschling, M.; Stamatakis, A.; Nindl, I.; Stockfleth, E.; Alonso, Á.; Bravo, I.G. Multiple Evolutionary Mechanisms Drive Papillomavirus Diversification. Mol. Biol. Evol. 2007, 24, 1242-1258. [CrossRef] [PubMed]

13. Gottschling, M.; Göker, M.; Stamatakis, A.; Bininda-Emonds, O.R.; Nindl, I.; Bravo, I.G. Quantifying the Phylodynamic Forces Driving Papillomavirus Evolution. Mol. Biol. Evol. 2011, 28, 2101-2113. [CrossRef] [PubMed]

14. Larsen, B.B.; Cole, K.L.; Worobey, M. Ancient DNA provides evidence of 27,000-year-old papillomavirus infection and long-term codivergence with rodents. Virus Evol. 2018, 4, vey014. [CrossRef] [PubMed]

15. Shah, S.D.; Doorbar, J.; Goldstein, R.A. Analysis of Host-Parasite Incongruence in Papillomavirus Evolution Using Importance Sampling. Mol. Biol. Evol. 2010, 27, 1301-1314. [CrossRef] 
16. Pimenoff, V.N.; De Oliveira, C.M.; Bravo, I.G. Transmission between Archaic and Modern Human Ancestors during the Evolution of the Oncogenic Human Papillomavirus 16. Mol. Biol. Evol. 2017, 34, 4-19. [CrossRef]

17. Chen, Z.; DeSalle, R.; Schiffman, M.; Herrero, R.; Wood, C.E.; Ruiz, J.C.; Clifford, G.M.; Chan, P.K.S.; Burk, R.D. Niche adaptation and viral transmission of human papillomaviruses from archaic hominins to modern humans. PLoS Pathog. 2018, 14, e1007352. [CrossRef]

18. Chen, Z.; Ho, W.C.S.; Boon, S.S.; Law, P.T.Y.; Chan, M.C.W.; DeSalle, R.; Burk, R.D.; Chan, P.K.S. Ancient Evolution and Dispersion of Human Papillomavirus 58 Variants. J. Virol. 2017, 91, e01285-17. [CrossRef]

19. Chen, Z.; Long, T.; Wong, P.Y.; Ho, W.C.S.; Burk, R.D.; Chan, P.K.S. Non-human Primate Papillomaviruses Share Similar Evolutionary Histories and Niche Adaptation as the Human Counterparts. Front. Microbiol. 2019, 10, 2093. [CrossRef]

20. D'Arc, M.; Moreira, F.R.R.; Dias, C.A.; Souza, A.R.; Seuánez, H.N.; Soares, M.A.; Tavares, M.C.H.; Santos, A.F.A. The characterization of two novel neotropical primate papillomaviruses supports the ancient within-species diversity model. Virus Evol. 2020, 6, veaa036. [CrossRef]

21. Hirose, Y.; Onuki, M.; Tenjimbayashi, Y.; Yamaguchi-Naka, M.; Mori, S.; Tasaka, N.; Satoh, T.; Morisada, T.; Iwata, T.; Kiyono, T.; et al. Whole-Genome Analysis of Human Papillomavirus Type 16 Prevalent in Japanese Women with or without Cervical Lesions. Viruses 2019, 11, 350. [CrossRef]

22. Tenjimbayashi, Y.; Onuki, M.; Hirose, Y.; Mori, S.; Ishii, Y.; Takeuchi, T.; Tasaka, N.; Satoh, T.; Morisada, T.; Iwata, T.; et al Whole-genome analysis of human papillomavirus genotypes 52 and 58 isolated from Japanese women with cervical intraepithelial neoplasia and invasive cervical cancer. Infect. Agents Cancer 2017, 12, 44. [CrossRef]

23. Yamaguchi-Naka, M.; Onuki, M.; Tenjimbayashi, Y.; Hirose, Y.; Tasaka, N.; Satoh, T.; Morisada, T.; Iwata, T.; Sekizawa, A.; Matsumoto, K.; et al. Molecular epidemiology of human papillomavirus 18 infections in Japanese Women. Infect. Genet. Evol. 2020, 83, 104345. [CrossRef] [PubMed]

24. Jinam, T.A.; Kanzawa-Kiriyama, H.; Saitou, N. Human genetic diversity in the Japanese Archipelago: Dual structure and beyond. Genes Genet. Syst. 2015, 90, 147-152. [CrossRef] [PubMed]

25. Kaifu, Y.; Fujita, M. Fossil record of early modern humans in East Asia. Quat. Int. 2012, 248, 2-11. [CrossRef]

26. Japanese Archipelago Human Population Genetics Consortium. The history of human populations in the Japanese Archipelago inferred from genome-wide SNP data with a special reference to the Ainu and the Ryukyuan populations. J. Hum. Genet. 2012, 57, 787-795. [CrossRef]

27. Wang, C.-C.; Yeh, H.-Y.; Popov, A.N.; Zhang, H.-Q.; Matsumura, H.; Sirak, K.; Cheronet, O.; Kovalev, A.; Rohland, N.; Kim, A.M.; et al. Genomic insights into the formation of human populations in East Asia. Nature 2021, 591, 413-419. [CrossRef]

28. Kanzawa-Kiriyama, H.; Kryukov, K.; Jinam, T.A.; Hosomichi, K.; Saso, A.; Suwa, G.; Ueda, S.; Yoneda, M.; Tajima, A.; Shinoda, K.-I.; et al. A partial nuclear genome of the Jomons who lived 3000 years ago in Fukushima, Japan. J. Hum. Genet. 2017, 62, 213-221. [CrossRef]

29. Kanzawa-Kiriyama, H.; Jinam, T.A.; Kawai, Y.; Sato, T.; Hosomichi, K.; Tajima, A.; Adachi, N.; Matsumura, H.; Kryukov, K.; Saitou, N.; et al. Late Jomon male and female genome sequences from the Funadomari site in Hokkaido, Japan. Anthr. Sci. 2019, 127, 83-108. [CrossRef]

30. Hanihara, K. Dual structure model for the population history of the Japanese. Jpn. Rev. 1991, 2, 1-33.

31. Omoto, K.; Saitou, N. Genetic origins of the Japanese: A partial support for the dual structure hypothesis. Am. J. Phys. Anthropol. 1997, 102, 437-446. [CrossRef]

32. McBride, A.A. The papillomavirus E2 proteins. Virology 2013, 445, 57-79. [CrossRef]

33. Murakami, I.; Egawa, N.; Griffin, H.; Yin, W.; Kranjec, C.; Nakahara, T.; Kiyono, T.; Doorbar, J. Roles for E1-independent replication and E6-mediated p53 degradation during low-risk and high-risk human papillomavirus genome maintenance. PLoS Pathog. 2019, 15, e1007755. [CrossRef]

34. Lehtinen, M.; Hibma, M.; Stellato, G.; Kuoppala, T.; Paavonen, J. Human T Helper Cell Epitopes Overlap B Cell and Putative Cytotoxic T Cell Epitopes in the E2 Protein of Human Papillomavirus Type 16. Biochem. Biophys. Res. Commun. 1995, 209, 541-546. [CrossRef] [PubMed]

35. Kónya, J.; Geijersstam, V.A.; Yuan, F.; Dillner, J.; Stuber, G.; Eklund, C. Identification of a cytotoxic T-lymphocyte epitope in the human papillomavirus type 16 E2 protein. J. Gen. Virol. 1997, 78 Pt 10, 2615-2620. [CrossRef] [PubMed] 